\title{
Clinical Trial Registration — Looking Back and Moving Ahead
}

In 2005, the International Committee of Medical Journal Editors (ICMJE) initiated a policy requiring investigators to deposit information about trial design into an accepted clinical trials registry before the onset of patient enrollment. ${ }^{1}$ This policy aimed to ensure that information about the existence and design of clinically directive trials was publicly available, an ideal that leaders in evidence-based medicine have advocated for decades. $^{2}$ The policy precipitated much angst among research investigators and sponsors, who feared that registration would be burdensome and would stifle competition. Yet, the response to this policy has been overwhelming. The ICMJE promised to reevaluate the policy in 2 years after implementation. Here, we summarize that reevaluation, specifically commenting on registries that meet the policy requirements, the types of studies that require registration, and the registration of trial results. As is always the case, the ICMJE establishes policy only for the 12 member journals (a detailed description of the ICMJE and its purpose is available at www.icmje.org), but many other journals have adopted our initial trial registration recommendations, and we hope that they will also adopt the modifications discussed in this update.

The research community has embraced trial registration. Before the ICMJE policy, ClinicalTrials. gov, the largest trial registry at the time, contained 13,153 trials; this number climbed to 22,714 1 month after the policy went into effect. ${ }^{3}$ In April 2007 , the registry contained over 40,000 trials, with more than 200 new trial registrations occurring weekly (Zarin D: personal communication). The four other registries that meet the ICMJE criteria have also grown as scores of journals have adopted the ICMJE clinical trials registration policy. In response to burgeoning registration, many investigators, sponsors, and government agencies have asked the ICMJE to recognize their local registries as databases that meet the policy. Fortunately, the World Health Organization's (WHO) International Clinical Trial Registry Platform (ICTRP), which was nascent when the ICMJE began to require trial registration, has matured rapidly and provides options for those that desire a wider array of registries. The ICTRP has taken the first steps toward developing a network of pri- mary and partner registers that meet WHO-specified criteria. ${ }^{4}$ Primary registers are WHO-selected registers managed by not-for-profit entities that will accept registrations for any interventional trials, delete duplicate entries from their own register, and provide data directly to the WHO. Partner registers, which will be more numerous, will include registers that submit data to primary registers but limit their own register to trials in a restricted area (such as a specific disease, company, academic institution, or geographic region).

The ICMJE strongly supports the WHO's efforts, through the ICTRP, to develop a coordinated process for identifying, gathering, deduplicating, and searching trials from registries around the world, thus eventually providing a one-stop search portal for those seeking information about clinical trials. In addition to the five existing registries, the ICMJE will now also accept registration in any of the primary registers that participate in the WHO ICTRP. Because it is critical that trial registries are independent of for-profit interests, the ICMJE policy requires registration in a WHO primary register rather than solely in a partner register, since for-profit entities manage some partner registers. As previously, trial registration with missing or uninformative fields for the minimum data elements is inadequate. ${ }^{1}$

Initially, the ICMJE required registration of all clinically directive trials, which it defined as "any research project that prospectively assigns human subjects to intervention or comparison groups to study the cause-and-effect relationship between a medical intervention and a health outcome." In May 2005, the ICMJE clarified this definition to exclude preliminary trials designed to study pharmacokinetics or major unknown toxicity (phase 1 trials). ${ }^{5}$ However, the ICMJE recognizes the potential benefit of having information about preliminary trials in the public domain, because these studies can guide future research or signal safety concerns. Consequently, the ICMJE is expanding the definition of the types of trials that must be registered to include these preliminary trials and adopts the WHO's definition of clinical trial: "any research study that prospectively assigns human participants or groups of humans to one or more health-related interventions to evaluate the effects on health 
Key Summary Points

In addition to accepting registration in any of the five existing registries, the ICMJE will accept registration of clinical trials in any of the primary registers that participate in the WHO ICTRP. Registration in a partner register only is insufficient.

The ICMJE will begin to implement the WHO definition of clinical trials for all trials that begin enrollment on or after July 1,2008 . This definition states that a clinical trial is "any research study that prospectively assigns human participants or groups of humans to one or more health-related interventions to evaluate the effects on health outcomes."

The ICMJE will not consider results posted in the same clinical trials registry in which the primary registration resides to be previous publication if the results are presented in the form of a brief ( $<500$ words) structured abstract or table.

outcomes." ${ }^{4}$ Health-related interventions include any intervention used to modify a biomedical or health-related outcome (for example, drugs, surgical procedures, devices, behavioral treatments, dietary interventions, and process-of-care changes). Health outcomes include any biomedical or health-related measures obtained in patients or participants, including pharmacokinetic measures and adverse events. As previously, purely observational studies (those in which the assignment of the medical intervention is not at the discretion of the investigator) will not require registration. The ICMJE member journals will start to implement the expanded definition of clinically directive trials for all trials that begin enrollment on or after July 1, 2008. Those who are uncertain whether their trial meets the expanded ICMJE definition should err on the side of registration if they wish to seek publication in an ICMJE journal.

Over the time during which registration of trial methods has become common practice, several forces have begun advocating for registration of trial results. We recognize that the climate for results registration will probably change dramatically and unpredictably over coming years. For the present, the ICMJE will not consider results posted in the same primary clinical trials register in which the initial registration resides as previous publication if the results are presented in the form of a brief ( $<500$ words) structured abstract or table. The ICMJE favors a standard abstract format for results reporting, and the CONSORT (Consolidated Standards for the Reporting of Trials) group's forthcoming guidelines for abstracts related to trials may be one such op- tion. The ICMJE believes that parties interested in results registration should consider requiring the deposition of such an abstract in the registry 24 months after closure of data collection if results are not published in a peer-reviewed venue by that time. The registered abstract should either cite any related full, peer-reviewed publications or include a statement that indicates that the report has not yet been published in a peerreviewed journal. Researchers should be aware that editors may consider more detailed deposition of trial results in publicly available registries to be prior publication. When submitting a paper, authors should fully disclose to editors all posting in registries of results of the same or closely related work.

Three years ago, trials registration was the exception; now it is the rule. Registration facilitates the dissemination of information among clinicians, researchers, and patients, and it helps to assure trial participants that the information that accrues as a result of their altruism will become part of the public record. The WHO's global efforts toward comprehensive trials registration and the ICMJE's requirements for registration aim to increase public trust in medical science.

Christine Laine, M.D., M.P.H.
Senior Deputy Editor, Annals of Internal Medicine

Richard Horton, F.Med.Sci.

Editor, The Lancet

Catherine D. DeAngelis, M.D., M.P.H.

Editor-in-Chief, JAMA

Jeffrey M. Drazen, M.D.

Editor-in-Chief, New England Journal of Medicine

Frank A. Frizelle, M.B., Ch.B., M.Med.Sc.

Editor-in-Chief, The New Zealand Medical Journal

Fiona Godlee, M.B., B.Chir., B.Sc.

Editor-in-Chief, BMJ

Charlotte Haug, M.D., Ph.D., M.Sc.

Editor-in-Chief, Norwegian Medical Journal

Paul C. Hébert, M.D., M.H.Sc.

Editor-in-Chief, Canadian Medical Association Journal

Sheldon Kotzin, M.L.S.

Executive Editor, MEDLINE, National Library of Medicine

Ana Marusic, M.D., Ph.D.

Editor, Croatian Medical Journal

Peush Sahni, M.S., Ph.D.

Representative and Past President, World Association of Medical Editors 
Torben V. Schroeder, M.D., D.M.Sc.

Editor, Journal of the Danish Medical Association

Harold C. Sox, M.D.

Editor, Annals of Internal Medicine

Martin B. Van Der Weyden, M.D.

Editor, The Medical Journal of Australia

Freek W.A. Verheugt, M.D.

Executive Editor, Nederlands Tijdschrift voor Geneeskunde (Dutch Journal of Medicine)

Editor's note: This editorial is being published simultaneously in all ICMJE member journals.

Disclaimer: Dr. Sahni's affiliation as a representative and pas president of the World Association of Medical Editors (WAME) does not imply endorsement by WAME member journals that are not part of the ICMJE.

Potential financial conflicts of interest: Employment: Dr. Godlee was previously editorial director of Current Controlled Trials, which owns the ISRCTN (International Standard Randomised Controlled Trial Number) trials register. Mr. Kotzin is employed by the National Library of Medicine, which produces ClinicalTrials.gov; Mr. Kotzin is not responsible for activities or policies regarding ClinicalTrials.gov. Expert testimony: F. Godlee. Other: R. Horton (co-chair, WHO ICTRP Scientific Advisory Group); J.M. Drazen (member, WHO ICTRP Scientific Advisory Group); H.C. Sox (member, WHO ICTRP Scientific Advisory Group); M.B. Van Der Weyden (member, government advisory committee for the Australian and New Zealand Clinical Trials Registry).

This article (10.1056/NEJMe078110) was published at www. nejm.org on June 4,2007

1. Clinical trial registration: a statement from the International Committee of Medical Journal Editors. (Accessed June 7, 2007, at http://www.icmje.org/clin_trial.pdf.)

2. Simes RJ. Publication bias: the case for an international registry of clinical trials. J Clin Oncol 1986;4:1529-41.

3. Zarin DA, Tse T, Ide NC. Trial registration at ClinicalTrials. gov between May and October 2005. N Engl J Med 2005;353: 2779-87.

4. International Clinical Trials Registry Platform (ICTRP). Geneva: World Health Organization. (Accessed June 7, 2007, at http://www.who.int/ictrp/about/details/en/index.html.)

5. De Angelis C, Drazen JM, Frizelle FA, et al. Is this clinical trial fully registered? A statement from the International Committee of Medical Journal Editors. (Accessed June 7, 2007, at http://www.icmje.org/clin_trialup.htm.)

Copyright (๑) 2007 Massachusetts Medical Society. 